\title{
External Lower Lip
}

National Cancer Institute

\section{Source}

National Cancer Institute. External Lower Lip. NCI Thesaurus. Code C12222.

The external surface of the lower lip. 